\title{
The gating charge pathway of an epilepsy-associated potassium channel accommodates chemical ligands
}

\author{
Ping $\mathrm{Li}^{1,{ }^{*}}$, Zhuxi Chen ${ }^{2, *}$, Haiyan $\mathrm{Xu}^{1}$, Haifeng $\mathrm{Sun}^{2}$, Hao Li ${ }^{2}$, Hong $\mathrm{Liu}^{2}$, Huaiyu Yang ${ }^{2}$, Zhaobing Gao \\ Hualiang Jiang ${ }^{2}$, Min $\mathrm{Li}^{1,3}$
}

${ }^{I}$ CAS Key Laboratory of Receptor Research, Shanghai Institute of Materia Medica, Chinese Academy of Sciences, 555 Zuchongzhi Road, Shanghai 201203, China; ${ }^{2}$ State Key Laboratory of Drug Research, Shanghai Institute of Materia Medica, Chinese Academy of Sciences, 555 Zuchongzhi Road, Shanghai 201203, China; ${ }^{3}$ The Solomon H Snyder Department of Neuroscience, High Throughput Biology Center and Johns Hopkins Ion Channel Center, School of Medicine, Johns Hopkins University, Baltimore, MD 21205, USA

Voltage-gated potassium (Kv) channels derive their voltage sensitivity from movement of gating charges in voltage-sensor domains (VSDs). The gating charges translocate through a physical pathway in the VSD to open or close the channel. Previous studies showed that the gating charge pathways of Shaker and Kv1.2-2.1 chimeric channels are occluded, forming the structural basis for the focused electric field and gating charge transfer center. Here, we show that the gating charge pathway of the voltage-gated KCNQ2 potassium channel, activity reduction of which causes epilepsy, can accommodate various small molecule ligands. Combining mutagenesis, molecular simulation and electrophysiological recording, a binding model for the probe activator, ztz240, in the gating charge pathway was defined. This information was used to establish a docking-based virtual screening assay targeting the defined ligand-binding pocket. Nine activators with five new chemotypes were identified, and in vivo experiments showed that three ligands binding to the gating charge pathway exhibit significant anti-epilepsy activity. Identification of various novel activators by virtual screening targeting the pocket supports the presence of a ligand-binding site in the gating charge pathway. The capability of the gating charge pathway to accommodate small molecule ligands offers new insights into the gating charge pathway of the therapeutically relevant KCNQ2 channel.

Keywords: voltage-gated potassium channel; gating charge pathway; KCNQ2 activator; binding site; anti-epilepsy drug Cell Research (2013) 23:1106-1118. doi:10.1038/cr.2013.82; published online 25 June 2013

\section{Introduction}

Voltage-gated potassium $(\mathrm{Kv})$ channels represent the largest group of potassium channels and play crucial roles in a wide range of physiological processes $[1,2]$. $\mathrm{Kv}$ channels constitute potential drug targets for the treatment of diverse diseases ranging from cancers to metabolic, cardiovascular and neurological disorders [3]. The

\footnotetext{
*These two authors contributed equally to this work. Correspondence: Hualiang Jiang ${ }^{\mathrm{a}}$, Zhaobing Gao ${ }^{\mathrm{b}}$, Huaiyu Yang ${ }^{\mathrm{c}}$ ${ }^{\mathrm{a}}$ Tel: +86-21-50805873; Fax: +86-21-50807088

E-mail: hljiang@mail.shcnc.ac.cn

${ }^{\mathrm{b}}$ Tel: +86-21-20239067; Fax: +86-21-20239067

E-mail: zbgao@simm.ac.cn

'Tel: +86-21-50800619; Fax: +86-21-50807088

E-mail: hyyang@simm.ac.cn

Received 12 January 2013; revised 16 April 2013; accepted 9 May 2013; published online 25 June 2013
}

six transmembrane segments of $\mathrm{Kv}$ channels are grouped into the voltage-sensor domain (VSD) (S1-S4) and the pore domain (S5-P-S6). The voltage-sensing function of the VSD is conferred by charged residues, mostly arginines in S4. To open or close the channel, these charged residues translocate between the internal and external water-accessible crevices in the VSD through a physical gating charge pathway in response to membrane potential changes [4]. Studies on Shaker and Kv1.2-2.1 chimeric channels suggest that the pathway is occluded in the middle of the VSD [5-11]. A conserved phenylalanine in the middle of S2 (F290 of the Shaker channel and F233 of the Kv1.2-2.1 chimera channel), together with the nearby arginines of S4, was proposed to form the occluded site $[5,7,12]$. The phenylalanine separates the extracellular and intracellular hydrated crevices of the VSD and forms the charge-transfer center that catalyzes movement of the gating charges $[5,13]$. Substitutions of 
certain arginines of Shaker S4 by smaller amino acids create an omega pore in the mutated VSD, which is permeable to some cations (e.g., $\mathrm{K}^{+}, \mathrm{Li}^{+}$and $\mathrm{Cs}^{+}$) $[7,12,14]$. The omega pore has been demonstrated to be the same as the gating charge pathway [5], which has the proper size to accommodate an arginine side chain [15]. The occluded pathway is consistent with the focused electric field theory, i.e., the transmembrane electric field is focused on the occluded site by the internal and external water crevices $[7,16]$. In addition, several previous studies showed that the arginines along S4 are accessible to methanethiosulfonate reagents $[8,17,18]$, implying that small molecules might bind to this site. The compounds ICA-27243 and NH29 were also suggested to act on the VSD $[19,20]$. These studies shed light on the central structures of the gating charge pathways of the Shaker channel and the Kv1.2-2.1 chimeric channel, and raised the possibility of the gating charge pathway as a therapeutic target $[5,13]$.

KCNQ2 potassium channel (Kv7.2) is a low-threshold $\mathrm{Kv}$ channel. Opening of KCNQ2 channel or heteromultimeric KCNQ2 complexes below threshold will inhibit initiation of action potentials, and thus KCNQ2 plays a fundamental role in controlling neuronal excitability [21]. Mutations of human $K C N Q 2$ genes that result in reduction or loss of channel activity cause benign familial neonatal convulsions. Activators capable of augmenting KCNQ2 function have been proven effective in treatment of human epilepsy [22]. In this study, we have identified an activator-binding pocket in the gating charge pathway of KCNQ2 by comprehensively employing homology modeling, molecular docking, molecular dynamics (MD) simulation, mutagenesis and electrophysiological determination. The small molecular activator that we recently discovered, ztz240 [23], was used as a probe for determining the binding model of ligands in the gating charge pathway. zt240 and two of the nine newly identified activators targeting the gating charge pathway exhibited comparable anticonvulsant activity as retigabine, a KCNQ activator approved as an anti-epilepsy drug in 2011 [24]. Our results offer insights into the gating charge pathway of KCNQ2 channel and demonstrate that the pathway can serve as a drug target for development of new therapeutics.

\section{Results}

Essential roles of the conserved phenylalanine (F137) for ztz240 activity

Compound ztz240 is a KCNQ2 activator identified recently [23]. Major effects of ztz240 on KCNQ2 channel include increasing outward current amplitude, left- shifting the voltage-dependent activation curve $(G-V$ curve) and dramatic slowing of deactivation (Figure 1). The phenylalanine forming the occluded site in Shaker and Kv1.2-2.1 chimeric channels is highly conserved in the family of $\mathrm{Kv}$ channels with the degree of conservation of $\sim 99 \%$ [25]. The corresponding conserved phenylalanine in KCNQ2 is F137, which is located around the suggested occluded site of gating charge pathway (Figure 1A and 1B; Supplementary information, Figure S1A). The activator ztz240 is mainly composed of two aromatic rings with hydrophobic properties linked via an amide bridge; we therefore speculated that it may form hydrophobic interactions with F137 if the compound could access this residue. To test this idea, we performed mutagenesis on the conserved residue. Indeed, mutation of KCNQ2 F137 to alanine (F137A) dramatically reduced ztz240 activity (Figure 1C-1F). The right-shifted dose-response curve of KCNQ2 F137A further supports the critical role of F137 for the activity of ztz240 (Figure $1 \mathrm{G})$. For the wild-type channel, the dose-response curve was a well-fit sigmoidal-shaped curve with an $\mathrm{EC}_{50}$ value of $2.81 \mu \mathrm{M}$. In contrast, the dose-response curve of KCNQ2 F137A could not be fit well, as potentiation is rather low even at higher concentrations.

One possible cause of the reduced sensitivity is that the transition into or out of open state of KCNQ2 channel is affected by F137A. However, negligible changing of biophysical parameters argues against F137A perturbing "state transition". The tested biophysical parameters of KCNQ2 F137A, such as current density, half maximal activation voltage $\left(V_{1 / 2}\right)$, time constants of activation (closed to open transition) and deactivation (open to closed transition), are very similar to those of wildtype channel (Supplementary information, Figure S2A). In addition, F137A did not reduce the activities of other KCNQ2 activators. Retigabine, an activator recognizing a tryptophan in the S5 segment [26, 27], was tested first. KCNQ2 F137A exhibited similar sensitivity to $10 \mu \mathrm{M}$ retigabine to wild-type channel (Supplementary information, Figure S2B). Next, ZnPy, another activator potentiating KCNQ channels through different mechanism from retigabine [28], was found to potentiate wild-type and F137A KCNQ2 channels in comparable scales (Supplementary information, Figure S2C). NH29, the third activator reported to target the external part of the VSD [20], also potentiated wild-type and F137A KCNQ2 channels in a similar manner (Supplementary information, Figure S2D). These data indicate that F137 is specifically critical for activity of ztz240.

Identification of more critical residues in the KCNQ2 VSD F137 is located at the middle of the S2 segment of 


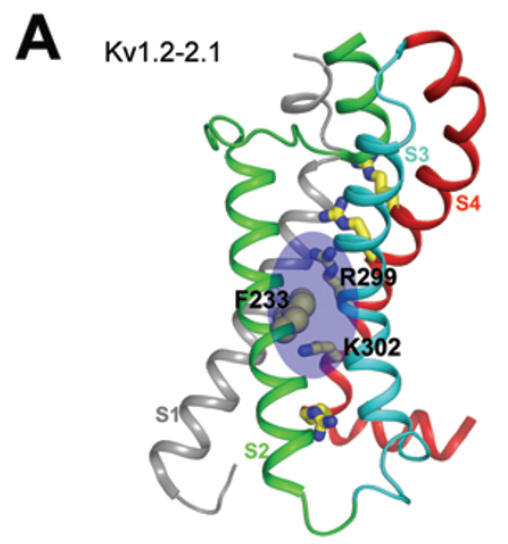

B
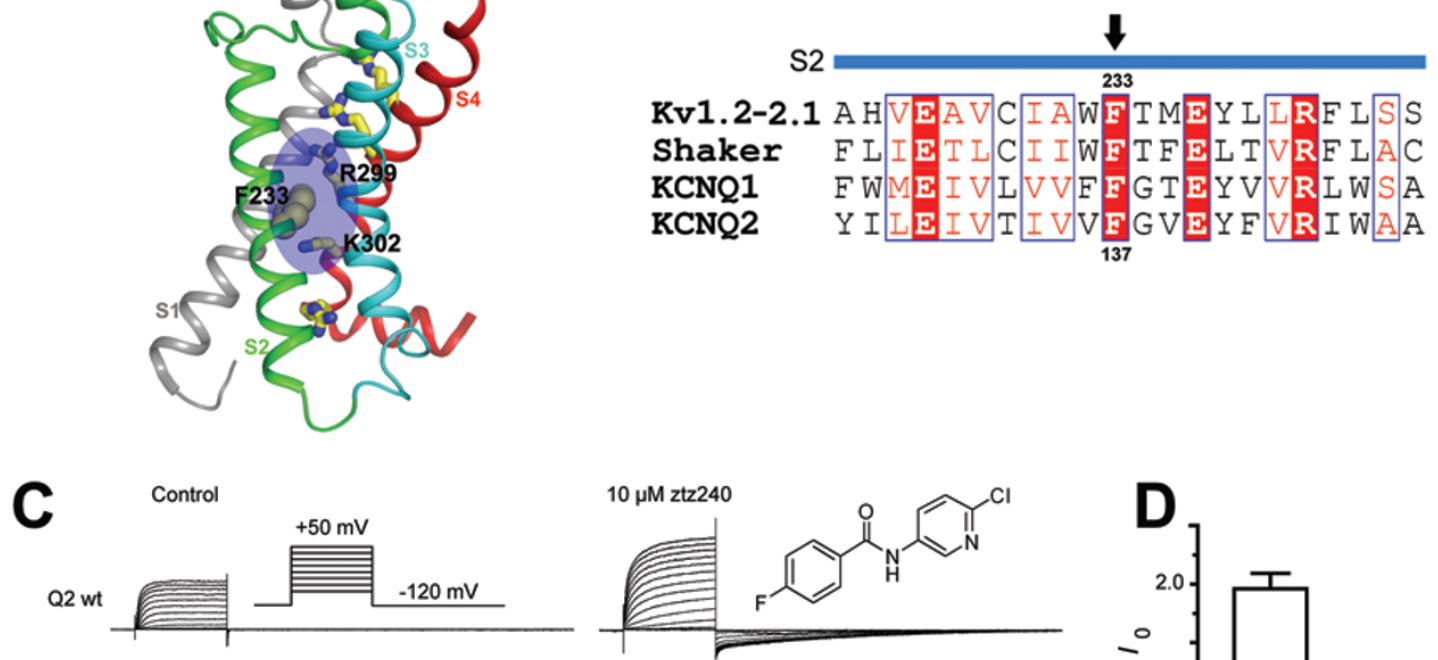

F137A
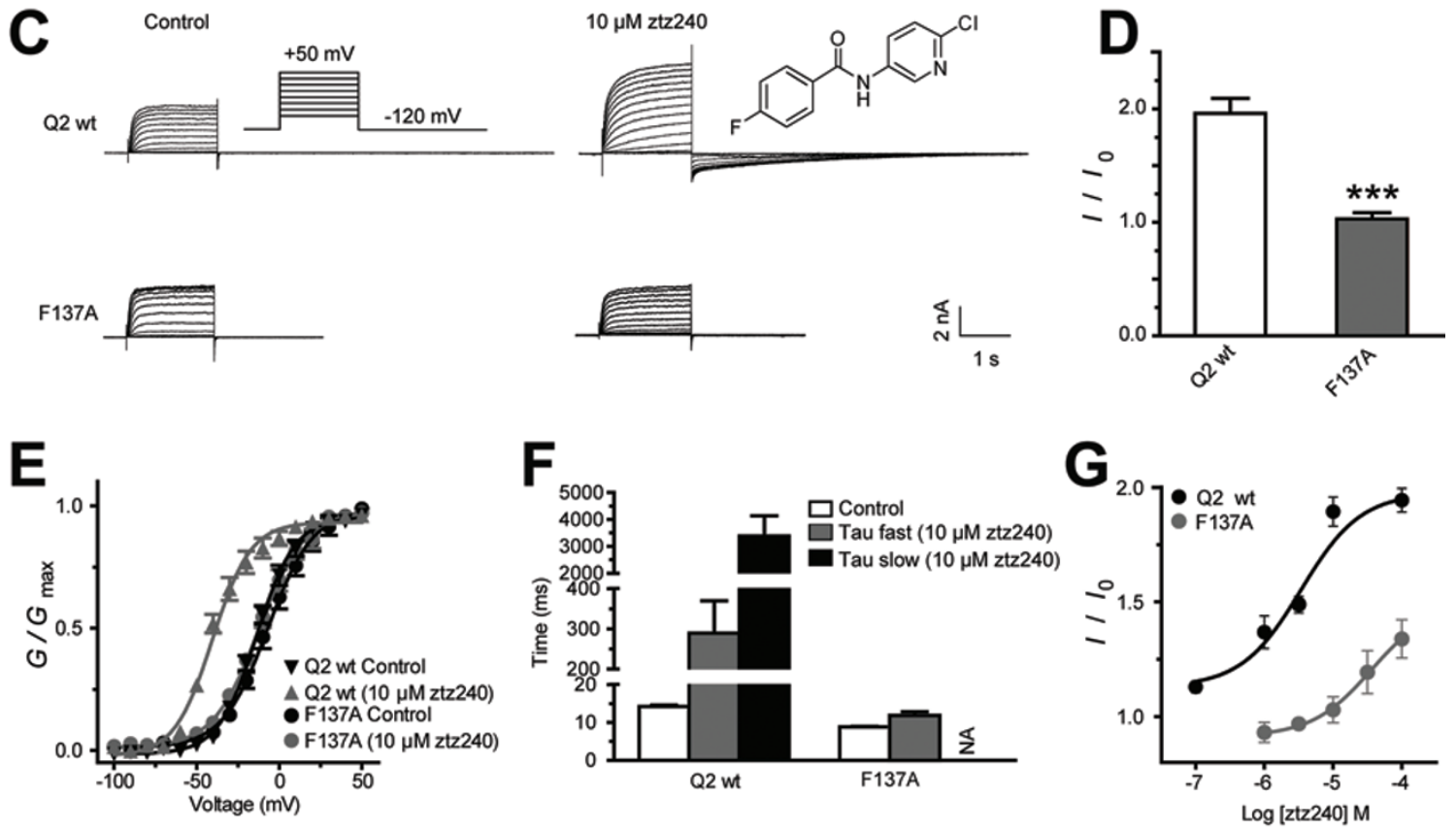

Figure $1 \mathrm{~F} 137$ of KCNQ2 is critical for ztz240 activity. (A) The crystal structure of the VSD of Kv1.2-2.1 chimeric channel (PDB code: 2R9R). The conserved phenylalanine in S2 (F233) is represented by spheres. Charged residues in S4 are displayed as sticks. The blue shadow indicates the occluded site. (B) Sequence alignment for S2. The conserved residues are highlighted in red. The conserved phenylalanine in S2 is indicated by an arrow. (C) Representative traces of wild-type and F137A KCNQ2 channels with or without $10 \mu \mathrm{M}$ ztz240. The inset shows the chemical structure of ztz240. (D) $10 \mu \mathrm{M}$ ztz240 potentiated outward current amplitude of wild-type, but not F137A KCNQ2 channels. The measured currents were elicited by $+50 \mathrm{mV}$. (E) G-V curves of wild-type and F137A KCNQ2 channels with or without ztz240. For wild-type KCNQ2, $10 \mu \mathrm{M}$ ztz240 leftshifted the $V_{1 / 2}$ dramatically, whereas the change for KCNQ2 F137A was negligible. (F) Histograms show the time constants of deactivation of wild-type and F137A KCNQ2 channels with or without ztz240. Deactivation phases that follow $+50 \mathrm{mV}$ depolarization were analyzed. For wild type, the deactivation phase was fit by a single exponential function before application of ztz240. However, the prolonged deactivation phase after application of ztz240 was fit by a biexponential function with fast $\left(\mathrm{Tau}_{\text {fast }}\right)$ and slow $\left(\mathrm{Tau}_{\text {slow }}\right)$ time constants. For KCNQ2 F137A, both the deactivation phases with and without ztz240 were fit well by a single exponential function. (G) Dose-response curve of ztz240 on wild-type and F137A KCNQ2 channels. The test potential is $+50 \mathrm{mV}$.

KCNQ2 VSD. We designed more mutants for the residues lining the VSD. Among the functional mutations, E130A, I134A, G138A, R207W and R210A dramatically decreased the potentiation activity of ztz240 in all three major aspects. They reduced the increase of outward current amplitude ratio $\left(I / I_{0}\right)$, and prevented or attenuated the left-shifting of the $G-V$ curve $\left(\Delta V_{1 / 2}\right)$ and the slowing of deactivation (Figure 2). The almost overlapping tail 
currents of these mutants with and without ztz240 clearly show the decreased effects on deactivation (Supplementary information, Table S1). In contrast, mutations S105A and M174A attenuated the left-shifting of $G-V$ curve but did not decrease $I / I_{0}$ ratio or prevent the slowing of deactivation (Figure 2 and Supplementary information, Table S1). Identification of multiple essential residues further indicates the importance of the VSD for ztz240 activity.

\section{Structural model of ztz240 binding to the KCNQ2 VSD}

As the experimental structures of KCNQ2 and its complexes with other ligands have not been determined, it is challenging to construct a structural model for the ztz240-KCNQ2 interaction. Accordingly, a hierarchical strategy was used to construct the structural model by comprehensively employing homology modeling, molecular docking and MD simulation in conjunction with mutagenesis and electrophysiological determinations. The mutagenesis result suggests that the potential binding pocket of ztz240 is mostly located in the VSD rather than in other domains (Figure 1). Further electrophysiological experiments support that ztz240 binds to the open-state VSD (Supplementary information, Figure S3). Thus, we first built up a three-dimensional (3D) model for the transmembrane domain of KCNQ2 based on the structural information of the open-state Kv1.2 channel [29-31] by using Discovery studio 2.6 (Supplementary information, Figure S1B).

Second, we constructed the binding model for ztz240 to KCNQ2. We defined a potential binding pocket for ztz240 according to the mutagenesis and electrophysiological data, which includes F137 and other residues essential for ztz240 to potentiate the channel (Figure 2 and Supplementary information, Table S1). Homology modeling could not predict proper conformations for the residues interacting with a ligand. Therefore, Induced Fit, a flexible docking program that considers the flexibilities of both ligand and side chains of a target protein, was applied to simulate the binding of ztz240 to KCNQ2. However, the current docking methods could not determine the orientation of ztz240 entering into the binding pocket, indicating that either end may enter into the pocket first (Supplementary information, Figure S4A). The orientation of ztz240 in the VSD was determined with the assistance of two derivatives of the compound (named YGP1 and YGP2) that have the fluorine and the chlorine substituted by hydrogen atoms, respectively. Electrophysiological recording showed that YGP1 still exhibited potentiation activity on KCNQ2 as ztz240 did; however, the potentiation activity of YGP2 was dramatically reduced (Supplementary information, Figure S4B and S4C). This result indicates that ztz240 may bind to the VSD via the configuration with chloropyridine forming the bottom of the pocket and the fluorophenyl located
A
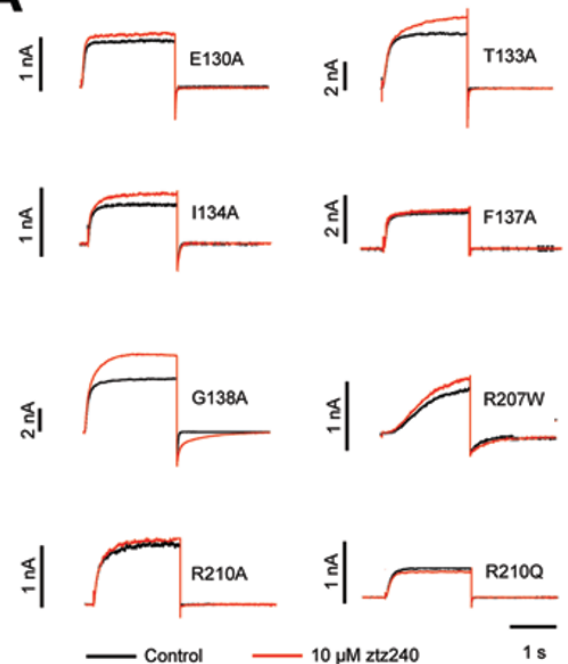

B

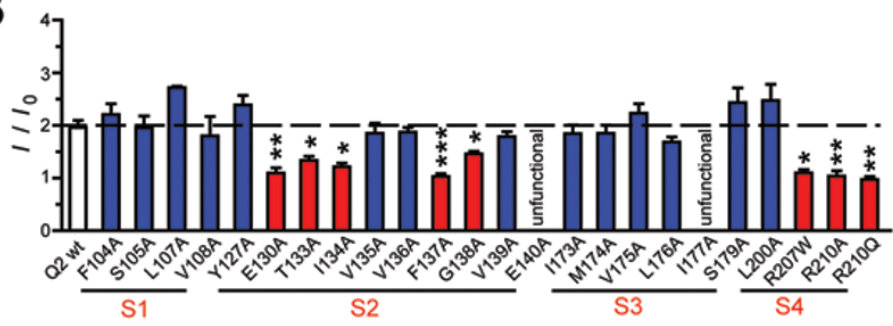

C

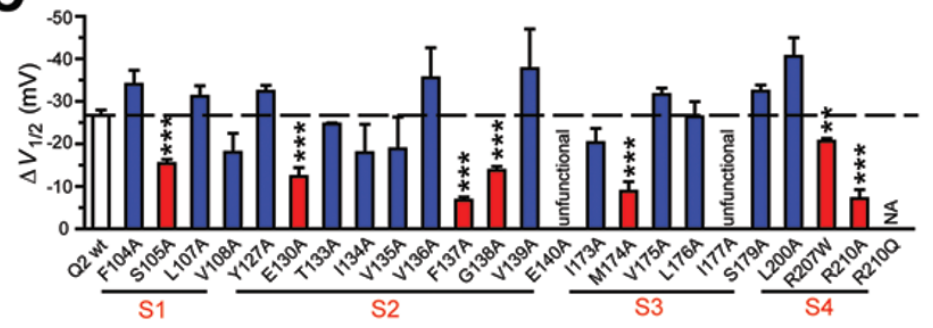

Figure 2 Identification of more critical residues in KCNQ2 VSD for ztz240 activity. (A) Representative traces of identified critical mutants with and without $10 \mu \mathrm{M}$ ztz240. (B) Effects of $10 \mu \mathrm{M}$ ztz240 on outward current amplitude of VSD mutants ( $n>$ 3). Each mutation site is indicated based on the predicted transmembrane regions. The dashed line indicates a potentiation level of $10 \mu \mathrm{M}$ ztz240 on the wild-type KCNQ2 channel. The test potential is $+50 \mathrm{mV}$. (C) Effects of $10 \mu \mathrm{M}$ ztz240 on $V_{1 / 2}$ of VSD mutants $(n>3)$. $\Delta V_{1 / 2}$ indicates the change of $V_{1 / 2}$ after application of ztz240. $\Delta V_{1 / 2}=V_{1 / 2}$ in the presence of $z$ tz240 - $V_{1 / 2}$ in control. 
near the pocket entrance (Supplementary information, Figure S4B).

Third, the ztz240-KCNQ2 binding model derived from the docking simulation was further optimized by MD simulation. The MD simulation may overcome the shortage of docking simulation that could not consider the flexibility of the protein backbone [32]. The model of the tetramer of ztz240/KCNQ2 complex was embedded into a patch of 1-palmitoyl-2-oleoyl-sn-glycero-3phosphocholine (POPC) bilayer and then subjected to a 200-ns MD simulation. The ligand-VSD interactions suggested by the optimized binding model are shown in Figure 3. ztz240 hydrophobically interacts with I134 and F137; the nitrogen atom of the linker forms hydrogen bonds with the side chain of E130; the pyridine ring is involved in a $\mathrm{CH}-\pi$ interaction with the $\alpha$-hydrogen of R207; the chlorine atom forms an electrostatic interaction with the guanidyl of R210 and a halogen bond with the carbonyl oxygen of R207, respectively; the pyridine nitrogen forms another electrostatic interaction with the guanidyl of R210. Residues that have been identified as critical for the potentiation activity of ztz240 in the mutagenesis studies are directly involved in the ztz240-VSD interactions, which explains well the mutagenesis data (Figure 2 and Supplementary information, Table S1).

Most of the crucial residues lining the binding pocket are located in S2 and S4, such as E130, I134, F137, G138, R207 and R210, while very few residues in S1 and S3 affect the compound, such as S105 and M174
(Figure 3), which are not conserved in KCNQ isoforms. To further evaluate the roles of residues in the external segments of S1 and S3 in ztz240 activation, the remaining residues except those listed in Figure 2B and 2C were examined by alanine scanning, and none of them significantly affected ztz240 activity (Supplementary information, Table S1). These additional mutations and electrophysiological determinations retrospectively validate again our ztz240-KCNQ2 binding model.

The model of the ztz240-KCNQ2 interaction clearly describes a broad pocket in the VSD, extending from the extracellular mouth of the VSD to R210 (Figure 3C). This possible ztz240-binding pocket in KCNQ2 partially overlaps with the corresponding regions of the gating charge pathways of Shaker and Kv1.2-2.1 chimeric channels, though it is more expansive and deeper (Figures $1 \mathrm{~A}$ and 3C) $[5-12,14,15]$. This result is consistent with previous studies indicating that the VSD of Kv channels might be druggable sites $[19,20]$. Our study further revealed that the gating charge pathway of KCNQ2 might have a new functionality, serving as a direct binding pocket for existing and newly designed activators such as ztz240 and new compounds discovered in this study (see below).

Discovering new activators targeting the ztz240-binding pocket

To discover new KCNQ2 activators and to further verify the possible activator-binding pocket, we carried out
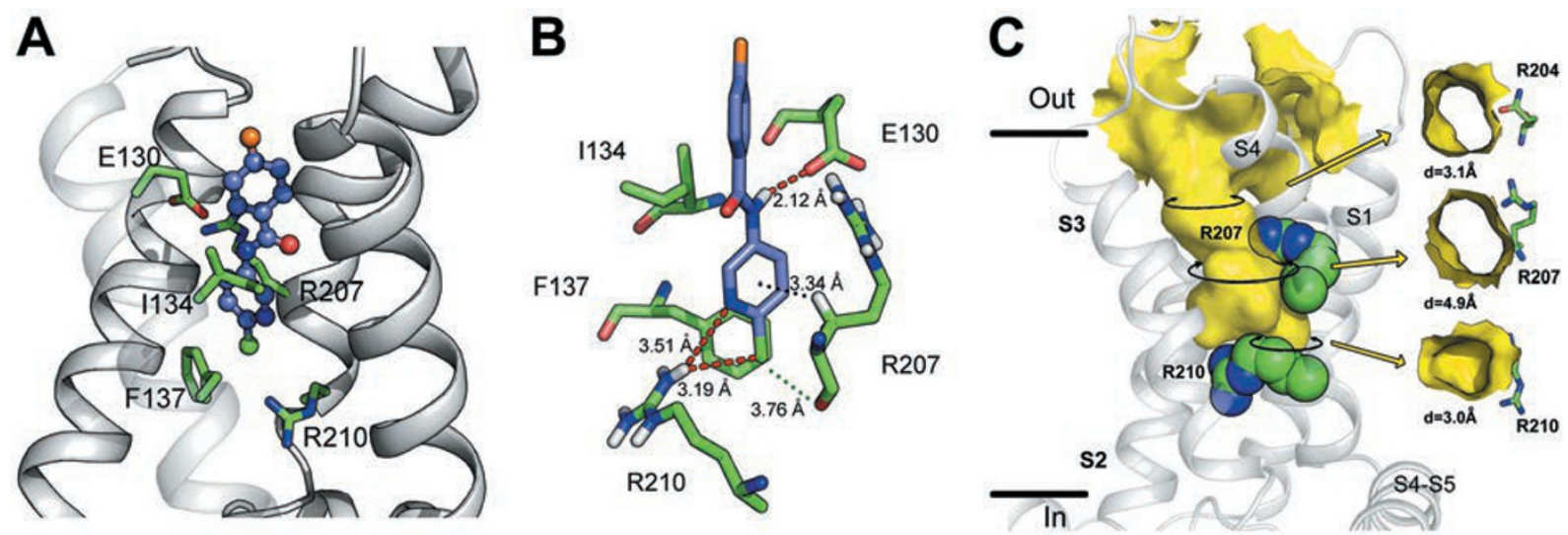

Figure 3 The binding model of ztz240 with VSD. (A, B) General (A) and detailed (B) views of the interactions between ztz240 and VSD. ztz240 and the residues are represented as ball-and-sticks. Carbon, oxygen, nitrogen, chlorine, fluorine and hydrogen atoms in ztz240 are colored in light blue, red, dark blue, green, orange and white, respectively. For clarity, only few key hydrogen atoms are shown. Hydrogen bonds or electrostatic interactions are indicated by red dashes. The $\mathrm{CH}-\pi$ interaction and halogen bond are indicated by black and green dashes, respectively. (C) The structure of the binding pocket extracted from the structural models of ligand-VSD complexes. For clarity, the ligands were removed. The surface of the pocket is shown in yellow. Three cross-sections of the pocket from the external view are shown on the right side. Residues are shown as sticks. 'Out' and 'In' indicate the external and the internal sides of cell membrane, respectively. 
a structure-based virtual screen targeting the identified pocket, guided by the notion that if the identified binding pocket is reasonable, novel activators recognizing the identified ztz240-binding pocket could be discovered. The SPECS database containing 200000 chemicals was screened by docking approach. Twenty-five hits (YG001YG025) were selected for bioassay. Nine compounds belonging to five different chemotypes displayed significant potentiation effects on the outward current of KCNQ2 channel (Figure 4). The potentiation ranges from $1.28 \pm$ 0.06 to $3.56 \pm 0.23$ at $30 \mu \mathrm{M}$ (Figure $4 \mathrm{~A}$ ). $\mathrm{EC}_{50}$ values of the three most potent active compounds, YG002, YG007 and YG025, were further revealed to be $4.64 \pm 0.14 \mu \mathrm{M}$, $1.60 \pm 0.16 \mu \mathrm{M}$ and $5.92 \pm 0.21 \mu \mathrm{M}$, respectively (Figure $4 \mathrm{~B}$ ). The $\mu \mathrm{M}$-level $\mathrm{EC}_{50}$ is similar to the approved drug retigabine [27]. The scaffolds of the nine identified activators can be described as two aryl groups connected by a functional linker. The linkers can be divided into five chemotypes, thiourea (YG002 and YG014), hydrazide (YG006, YG009 and YG025), urea (YG007 and YG018), amide (YG021) and amine (YG023). YG021 is an analogue of the reported activator ICA-27243 [33], but the other four chemotypes have never been reported before as KCNQ activators. The key residues involved in the interaction of ztz240 with the VSD, such as F137 and R210, are still essential for the newly identified activators (Figure 4D). Consistently, both F137A and R210A mutations were found to differentially affect the activity of most of these newly identified ligands (Supplementary information, Figure S5). This result demonstrates again that the gating charge pathway of KCNQ2 may accommodate various activators.

Anti-epilepsy activity of the activators targeting the gating charge pathway

A primary anti-epilepsy screening assay was per-
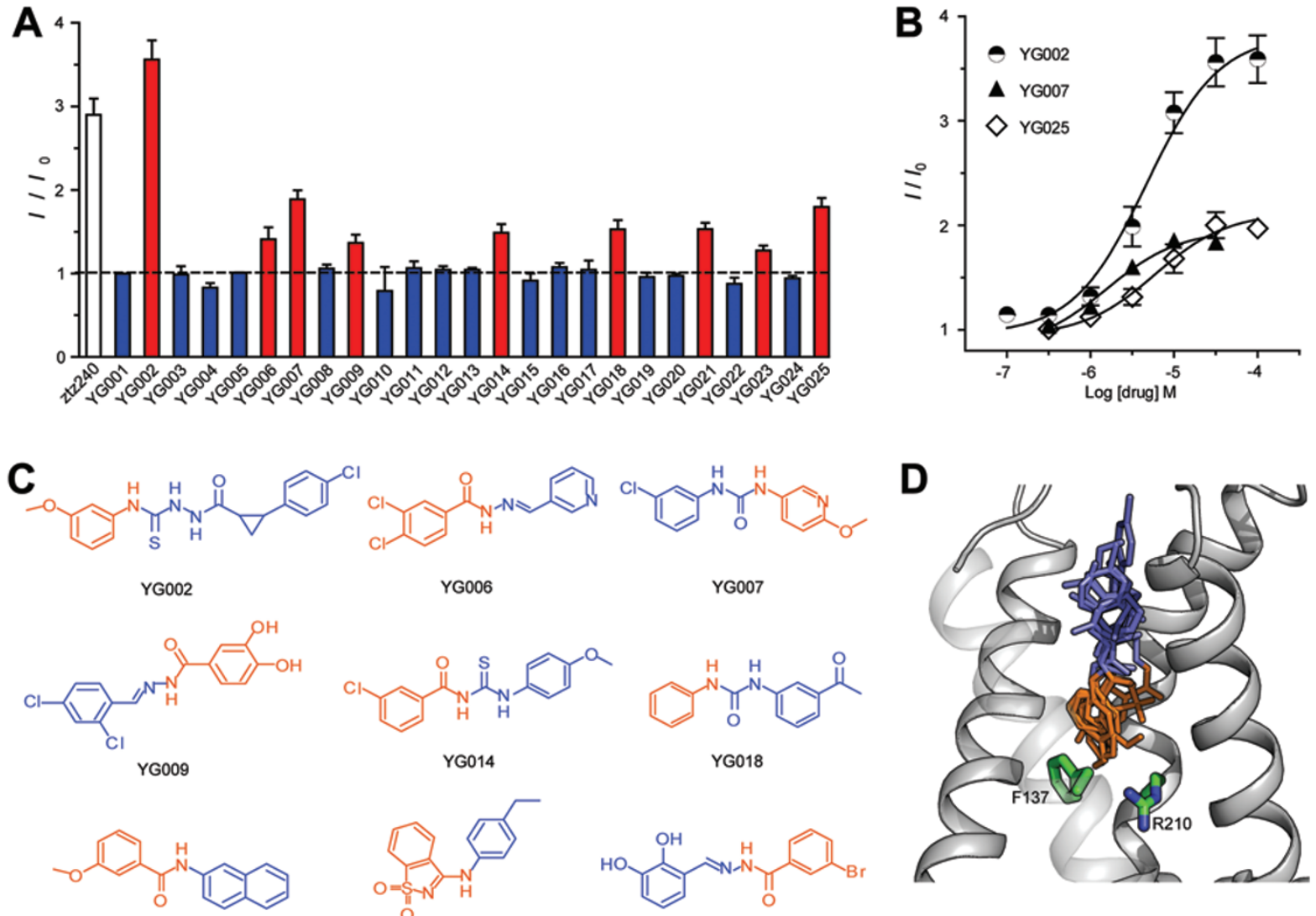

YG021
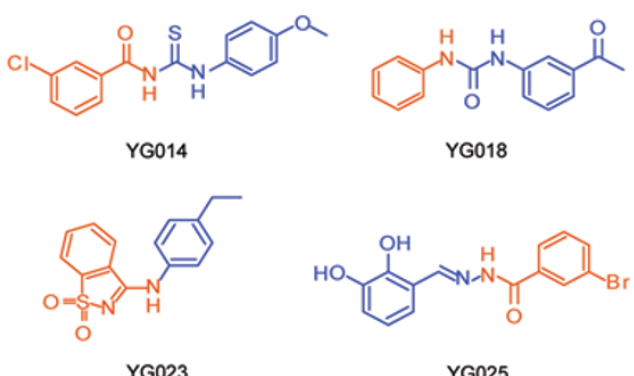

Figure 4 Structure-based virtual screening identifies activators with diverse chemotypes. (A) The increase of the outward current amplitude in the presence of compound as indicated. The test potential is $-10 \mathrm{mV}$. The dashed line indicates a potentiation level of 1 (i.e., no potentiation). (B) Dose-response curves of YG002, YG025 and YG027 ( $n>3$ for each data point). (C) Chemical structures of the discovered activators. The fragments oriented toward the intracellular end of the VSD are highlighted in orange. (D) General view of superimposed binding models of nine identified activators with the VSD. 
Table 1 Effects of ztz240, YG007 and YG018 on mouse MESinduced seizure model

\begin{tabular}{lccc}
\hline Group & Administration & Episodes of seizure & Protection (\%) \\
\hline YG007 & $40 \mathrm{mg} / \mathrm{kg}$ & $1 / 10^{* * *}$ & $90^{* * *}$ \\
YG018 & $40 \mathrm{mg} / \mathrm{kg}$ & $1 / 10^{* * *}$ & $90^{* * *}$ \\
ztz240 & $20 \mathrm{mg} / \mathrm{kg}$ & $0 / 8^{* * *}$ & $100^{* * *}$ \\
Retigabine & $20 \mathrm{mg} / \mathrm{kg}$ & $0 / 8^{* * *}$ & $100^{* * *}$ \\
Vehicle & $0.2 \mathrm{ml} / 10 \mathrm{~g}$ & $10 / 10$ & 0 \\
\hline
\end{tabular}

Abbreviation: MES, maximal electroshock. ${ }^{* * *} P<0.001$, significantly different from vehicle, Fisher's exact probability test.

formed to evaluate the activity of these compounds in mice using the maximal electroshock (MES)-induced seizure model. Among the tested activators, ztz240, YG007 and YG018 exhibited $>50 \%$ protection rate and thus were selected for further investigation in MESinduced and pentylenetetrazol (PTZ)-induced seizure models (Supplementary information, Table S2). Retigabine was set as the positive control. All three compounds exhibited excellent anti-epilepsy activity in both models. In the MES-induced seizure model, a single dose administration of the three compounds significantly prevented the tonic hind limb extension. The protection rates $(100 \%$, $90 \%$ and $90 \%$ ) are comparable with that of retigabine (Table 1). In the PTZ-induced seizure model, the three compounds exhibited comparable anti-epilepsy activity as retigabine. A single dose of these compounds not only significantly delayed the clonus latency but also reduced the incidence of generalized tonic-clonic seizure (GTCS) and mortality (Table 2).

\section{Discussion}

For many drug targets, such as GPCRs, nuclear receptors and ligand-gated ion channels, their functions are modulated by endogenous ligands. Accordingly, many drugs bind receptors at sites where the natural molecular activators (i.e., endogenous ligands) act [34]. In contrast, the binding site of $\mathrm{Kv}$ channel activators is more elusive, as the activation of $\mathrm{Kv}$ channels in the physiological environment is modulated by membrane potential rather than endogenous ligands. Elucidation of the binding of activators with $\mathrm{Kv}$ channels is essential both for understanding the mechanism of channel activation and for the development of therapeutics. To date, limited information about the binding site of activators of $\mathrm{Kv}$ channels is available. The present study provides evidence for the presence of a ligand-binding site in the gating charge pathway of KCNQ2 channel. The identified activatorbinding site lying deeply inside the VSD is largely different from postulated sites reported previously, which are either located at the pore region or a superficial part of the VSD [20, 26, 27]. The newly identified site encases the activators through multiple transmembrane segments.

To characterize a ligand-binding site, crystallography of the ligand/protein complex is the most direct method. However, membrane proteins are notoriously difficult to purify and crystallize. Very few $\mathrm{Kv}$ channels have been crystallized successfully [35-37], and no drug/Kv complex structure has been determined. Radioactive ligandbinding assays or photo-labeling experiments have not answered the question either. As a primary strategy for exploring the action sites of activators, mutagenesis experiments have been effective in identifying the key molecular determinants for potentiation on hERG and KCNQ channels [38-42]. However, the limitation of mutagenesis is the inability to exclude a binding site or a key site that is only essential for the activity of the compound, but not for binding. Our study provides an alternative way to distinguish binding sites from other key sites for channel modulators. We speculated that new activators would be found based on the 'binding site' by structure-based drug discovery if the 'binding site' is true. Structure-based virtual screening has been widely applied in drug discovery. A virtual screen of 200000 compounds in the SPECS database targeting this binding site was performed. Twenty-five hits were selected for biological validation and nine showed potentiation on KCNQ2 channel in the voltage clamp assay, which is considered as the 'gold standard' for the study of ion

Table 2 Effects of ztz240, YG007 and YG018 on mouse PTZ-induced seizure model

\begin{tabular}{|c|c|c|c|c|}
\hline Group & Administration & Clonus latency (s) & Incidence of GTCS & Mortality \\
\hline YG007 & $40 \mathrm{mg} / \mathrm{kg}$ & $161 \pm 42^{*}$ & $3 / 10^{*}$ & $5 / 10^{*}$ \\
\hline YG018 & $40 \mathrm{mg} / \mathrm{kg}$ & $191 \pm 27^{* * *}$ & $1 / 10^{* *}$ & $2 / 10^{* * *}$ \\
\hline Retigabine & $20 \mathrm{mg} / \mathrm{kg}$ & $181 \pm 40^{* * *}$ & $0 / 10^{* * *}$ & $2 / 10^{* * *}$ \\
\hline Vehicle & $0.2 \mathrm{ml} / 10 \mathrm{~g}$ & $116 \pm 26$ & $8 / 10$ & $10 / 10$ \\
\hline
\end{tabular}

Abbreviations: GTCS, generalized tonic-clonic seizure; PTZ, pentylenetetrazol. ${ }^{*} P<0.05,{ }^{* *} P<0.01,{ }^{* * *} P<0.001$, significantly different from vehicle, Fisher's exact probability test. 
channels. The hit rate of our virtual screening is $36 \%$ $(9 / 25)$, which is much higher than the hit rate $(0.1 \%)$ of a cell-based high throughput screening for discovering KCNQ2 activators (http://pubchem.ncbi.nlm.nih.gov). In addition, the fact that all identified novel activators recognized the same site further supports the presence of the ligand-binding site. It should be noted that the complex model was built based on a homology model of KCNQ2 channel, which may contain several inherent limitations. There is no representation of cation- $\pi$ interaction and halogen bond in empirical force fields. Molecular docking and MD simulation applied in the current work could not fully characterize these interactions. These particular interactions were proposed based on geometric considerations. Further crystallography or combined quantum mechanics/molecular mechanics (QM/MM) studies are necessary to fully elucidate the binding mode of ztz 240 in the binding pocket.

The occluded gating charge pathways of Shaker and Kv1.2-2.1 chimeric channels focus the electric field on a narrow region and form the charge-transfer center, both of which were suggested to be important for the mechanism of voltage gating of Kv channels. This study shows a new functionality of the gating charge pathway of KCNQ2, which serves as a direct binding pocket for activators. The broad pocket in the VSD extends from the extracellular mouth of the VSD to R210 (Figure 3C) and allows chemical ligands to enter and reside in the space beneath R207. The volume of this space is $\sim 170$ $\AA^{3}$, which is large enough to accommodate the aromatic rings of ztz240 and our newly identified ligands (moieties highlighted in orange in Figure 4D) with sizes of 90 to $140 \AA^{3}$. The ability of the KCNQ2 VSD to bind chemical ligands suggests that the gating charge pathway of the channel might be structurally different from other $\mathrm{KV}$ channels, such as the Kv1.2-2.1 chimera. The phenylalanine (F233) is the key residue of the charge-transfer center in the Kv1.2-2.1 chimera. When it was substituted by 19 other natural amino acids, the voltage dependence of most mutants was largely affected [13]. However, analogous mutations F137X (X represents the other 19 natural amino acids) cause much less influence on the voltage dependence of KCNQ2 channel gating (Supplementary information, Figure S6). First, all 19 KCNQ2 mutants are functional, whereas only $15 \mathrm{Kv} 1.2-2.1$ mutants are functional. When the phenylalanine is mutated to aspartic acid (D), lysine (K) or arginine (R), the KCNQ2 channels are still functional while the Kv1.2-2.1 channels lose functionality, suggesting that the phenylalanine (F137) in KCNQ2 is more tolerant to changes. Second, effects of the mutations on $G-V$ curves are different. Among the 15 functional Kv1.2-2.1 mutants, 13 exhibit largely right-shifted $G$ - $V$ curves with positive $V_{1 / 2}$ and the rightshifting effects are $>100 \mathrm{mV}$ for some mutants, such as F233A [13]. Only two mutations, tryptophan (W) and tyrosine (Y), exhibit currents near wild-type levels with negative $V_{1 / 2}$, which is consistent with the notion that a rigid cyclic side chain is important at this position [13]. In contrast, for KCNQ2, only six mutants display positive $V_{1 / 2}$ values and the right-shifting is $<100 \mathrm{mV}$ for all the mutations. Furthermore, besides F137W and F137Y, F137A, F137V, F137I and F137L also exhibit similar $V_{1 / 2}$ values to that of the wild type, suggesting that the cyclic side chain at F137 position is not as important as in the Kv1.2-2.1 chimera. However, for some specific substitutions, the mutations lead to similar kinetic effects for the two channels. For instance, both Kv1.2-2.1 F233W and KCNQ2 F137W exhibit a slower rate of closure than wild-type channels (Supplementary information, Figure S6C and S6D) [13]. The discordant phenotypes of the F137X and F233X mutants support the idea that gating charge pathways are different at this particular position. Other explanations for discordant phenotypes between KCNQ2 and Kv1.2-2.1 chimeric channels are possible. Interestingly, the present concept of diverse gating charge pathways is in agreement with the observation that the voltage-gated proton channel Hv1 forms an internal water wire penetrating the whole VSD [43-45]. The Hv1 channel only contains four transmembrane segments homologous to the VSD of Kv channels, but lacks the classic pore domain of other voltage-gated ion channels.

Although there is no available atomic-resolution structure of a Kv channel in the resting state, the first four arginines (R1-R4) of Kv1.2 are generally thought to stay in a 'down' position in the resting state compared with the open state [46, 47]. A recent long-time all-atom MD simulation also supports the downward movement of arginines [5]. The aromatic moieties of ztz240 and other identified activators reside in the gating charge pathway by filling the space between R207 and R210, thereby preventing the arginine from moving down to its resting state (Figure 5 and Supplementary information, Movie S1). The capability of the KCNQ2 VSD to accommodate chemical ligands allows the possibility to upregulate $\mathrm{Kv}$ channel function and treat diseases through acting at the gating charge pathway directly. This notion was examined by in vivo experiments. Anti-epilepsy effects of ztz240 and the nine newly identified activators were tested in the mouse MES-induced seizure model. Among them, ztz240, YG007 and YG018 exhibit anti-epilepsy activity and protection rates comparable to that of retigabine (Table 1). Different from ztz240, retigabine activates KCNQ2-5 through a crucial tryptophan residue in 

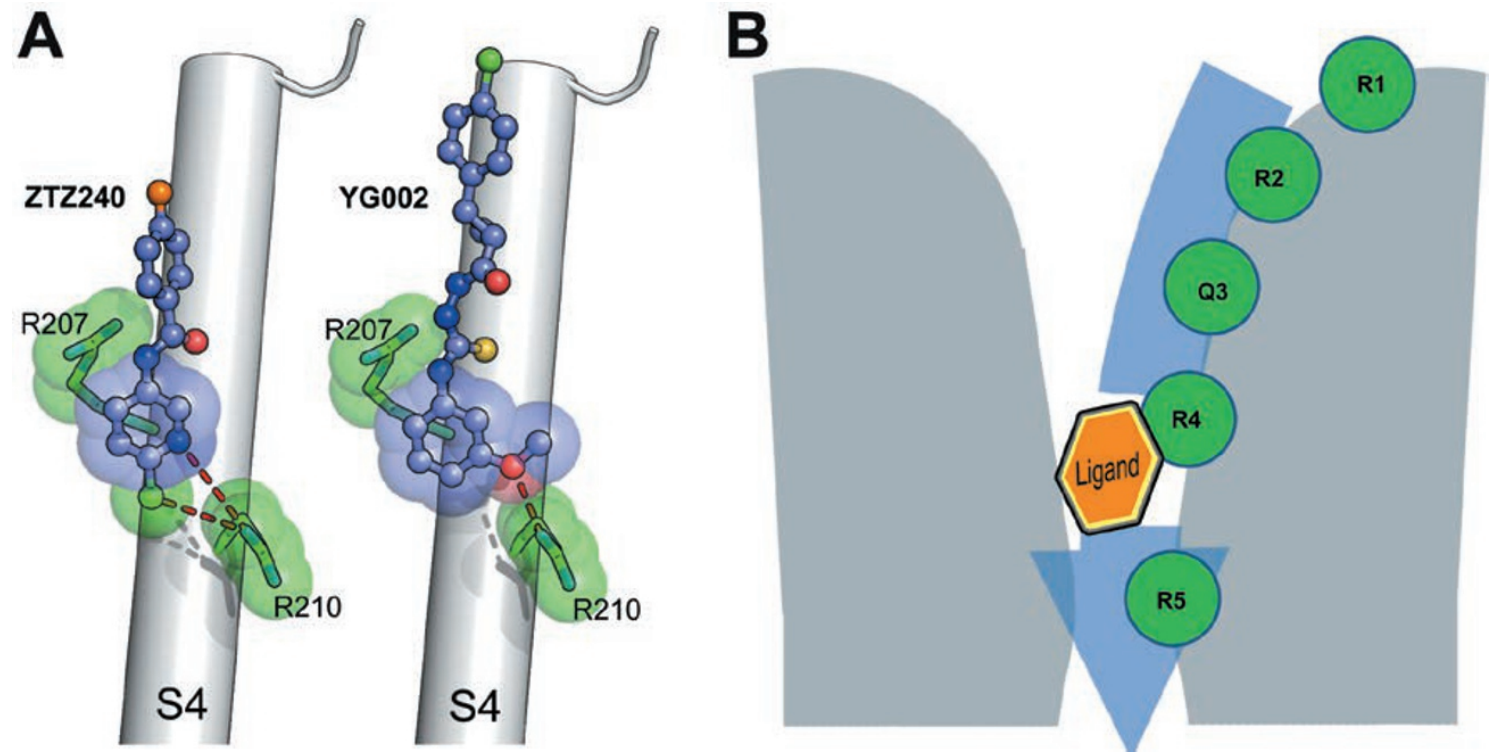

Figure 5 The gating charge pathway of KCNQ2 channel accommodates chemical ligands. (A) Details of how activators interact with and affect R207 and R210 in open state, using ztz240 and YG002 as examples. The activators fill the space between R207 and R210. The dashed lines indicate the interactions between the activators and R210. (B) The cartoon shows how a chemical ligand acts on the gating charge pathway by residing in the gating charge pathway and hindering the arginine to move to its down state.

S5 [26, 27]. As KCNQ channels are accessible to various types and combinations of pharmacological regulation [28], ztz240 and retigabine may lead to a synergistic effect, which might be beneficial for treatment of epilepsy. In summary, our study provides new insights into the structures and functions of gating charge pathways of $\mathrm{Kv}$ channels. The discovery of new modulators targeting the gating charge pathway by virtual screening may have general implications for other voltage-gated ion channels.

\section{Materials and Methods}

\section{Mutagenesis}

Starting from KCNQ cDNAs, the KCNQ2 point mutants were constructed by recombinant PCR and verified by sequencing.

\section{Cell culture and transient transfection}

Chinese Hamster Ovary (CHO) cells were grown in 50/50 DMEM/F12 (Cellgro, Manassas, VA, USA) with 10\% fetal bovine serum (FBS) and $2 \mathrm{mM}$ L-glutamine (Gibco, Carlsbad, CA, USA). To express ion channels, cells were split at $24 \mathrm{~h}$ before transfection, plated in 60-mm dishes and transfected with Lipofectamine $2000^{\mathrm{TM}}$ reagent (Invitrogen, Carlsbad, CA, USA), according to the manufacturer's instruction. At $24 \mathrm{~h}$ after transfection, cells were split and replated onto coverslips coated with poly-L-lysine (Sigma-Aldrich, St Louis, MO, USA). A plasmid for cDNA of GFP (Amaxa, Gaithersburg, MD, USA) was co-transfected to aid the identification of transfected cells by fluorescence microscopy.

\section{Electrophysiological recording}

Whole-cell voltage clamp recording was carried out at room temperature in $\mathrm{CHO}$ cells with an Axopatch-200B amplifier (Molecular Devices, Sunnyvale, CA, USA). The electrodes were pulled from borosilicate glass capillaries (World Precision Instruments, Sarasota, FL, USA). When filled with the intracellular solution, the electrodes had resistances of 3-5 M 2 . Pipette solution contained: $\mathrm{KCl} 145 \mathrm{mM}, \mathrm{MgCl}_{2} 1 \mathrm{mM}$, EGTA $5 \mathrm{mM}$, HEPES 10 $\mathrm{mM}$ and MgATP $5 \mathrm{mM}$ (pH 7.3 with $\mathrm{KOH})$. During the recording, constant perfusion of extracellular solution was maintained using a BPS perfusion system (ALA scientific Instruments, Westburg, NY, USA). Extracellular solution contained: $\mathrm{NaCl} 140 \mathrm{mM}, \mathrm{KCl} 3$ $\mathrm{mM}, \mathrm{CaCl}_{2} 2 \mathrm{mM}, \mathrm{MgCl}_{2} 1.5 \mathrm{mM}$, HEPES $10 \mathrm{mM}$ and glucose 10 $\mathrm{mM}(\mathrm{pH} 7.4$ with $\mathrm{NaOH})$. Signals were filtered at $1 \mathrm{kHz}$, and digitized using a DigiData 1440 with pClamp 9.2 software (Molecular Devices). Series resistance was compensated by $60 \%-80 \%$.

\section{Mouse MES- and PTZ-induced seizure models}

All animal procedures were performed in accordance with the National Institutes of Health Guide for the Care and Use of Laboratory Animals, under protocols approved and strictly followed by the Institutional Animal Care and Use Committees (IACUC). The IACUC checked all protocols and approved this study. Male KM mice, weighing 18-22 g, were obtained from Shanghai SLAC Laboratory Animal CO Ltd. For the MES-induced seizure model, the shock level was set at $160 \mathrm{~V}$ and the duration was set at $5.4 \mathrm{~s}$ with continuous wave under the eighth configuration, using a physiological and pharmacological electronic stimulator (Jinan, Shandong, China). The day before the experiment, the mice with GTCS induced by MES were prepared for the tests of the anticonvulsant effects of the compounds. Mice were separately administered with 
the testing compounds or vehicle in 5\% dimethylsulfoxide/95\% $(0.2 \%)$ hydroxymethyl cellulose by intraperitoneal injection 30 min before electroshock application in the mouse; the compound retigabine was used as the positive control. The incidence of hind limb extension was recorded, and if extension did not occur in the experiment, the animal was thought to be protected against the MES-induced seizure. For the PTZ-induced model, compounds were prepared in the same way as those in the MES-induced model and administered to male $\mathrm{KM}$ mice by intraperitoneal injection in a volume of $0.2 \mathrm{ml} / 10 \mathrm{~g} 30 \mathrm{~min}$ before administration of $100 \mathrm{mg} /$ $\mathrm{kg}$ PTZ sc. The latency to clonic seizure was recorded in seconds, and the incidence of GTCS and mortality rate caused by PTZ were both measured in this test.

\section{Test compounds}

Synthesis and characterization of YGP1 (N-(6-Chloropyridin3-yl)benzamide) To a solution of benzoic acid $(0.36 \mathrm{mmol})$ in 2 $\mathrm{ml}$ THF at $0{ }^{\circ} \mathrm{C}, N, N^{\prime}$-dicyclohexylcarbodiimide (DCC) was added with stirring for $30 \mathrm{~min}$. 6-chloropyridin-3-amine $(0.3 \mathrm{mmol})$ was then added to the mixture followed by addition of dimethyl aminopyridine (DMAP, $0.06 \mathrm{mmol}$ ) at room temperature with stirring overnight. After evaporating the volatiles, the mixture was partitioned by $\mathrm{CH}_{2} \mathrm{Cl}_{2}(2 \times 20 \mathrm{ml})$ and saturated with $\mathrm{NaHCO}_{3}$ solution $(20 \mathrm{ml})$, dried over anhydrous $\mathrm{Na}_{2} \mathrm{SO}_{4}$ and concentrated in vacuo. The residue was purified by column chromatography to give the compound YGP1 with 82\% yield. HPLC (Agilent 1100; column/ Extend-C18, $5 \mu \mathrm{m}, 4.6 \times 150 \mathrm{~mm}$; methanol:water/60:40; $0.6 \mathrm{ml} /$ $\min ),>98 \%$; ${ }^{1} \mathrm{H}$ NMR $\left(400 \mathrm{MHz}, \mathrm{CD}_{3} \mathrm{OD}\right) \delta 8.73-8.72(\mathrm{~m}, 1 \mathrm{H})$, 8.26-7.23 (m, 1H), 7.96-7.93 (m, 2H), 7.62-7.58 (m, 1H), 7.54-7.46 $(\mathrm{m}, 2 \mathrm{H}), 7.44(\mathrm{~d}, J=0.8 \mathrm{~Hz}, 1 \mathrm{H}) ;{ }^{13} \mathrm{C} \mathrm{NMR}\left(125 \mathrm{MHz}, \mathrm{CD}_{3} \mathrm{OD}\right)$ $\delta 167.54,145.11,141.35,135.28,134.05,131.95,131.26,128.34$, 127.35, 124.06; MS (ESI) m/z: $233[\mathrm{M}+\mathrm{H}]^{+}$; HRMS (ESI) 255.0301 Calcd for C12H9N2OCINa, found 255.0311 [M+Na] $]^{+}$.

Synthesis and characterization of YGP2 (4-Fluoro-N-(pyridin3-yl)benzamide) To a solution of 4-fluorobenzoic acid ( 0.36 $\mathrm{mmol}$ ) in $2 \mathrm{ml} \mathrm{THF}$ at $0{ }^{\circ} \mathrm{C}, \mathrm{DCC}$ was added with stirring for 30 min. Then, pyridin-3-amine $(0.3 \mathrm{mmol})$ was added to the mixture followed by addition of DMAP $(0.06 \mathrm{mmol})$ at room temperature with stirring overnight. After evaporating the volatiles, the mixture was partitioned by $\mathrm{CH}_{2} \mathrm{Cl}_{2}(2 \times 20 \mathrm{ml})$ and saturated with $\mathrm{NaHCO}_{3}$ solution $(20 \mathrm{ml})$, dried over anhydrous $\mathrm{Na}_{2} \mathrm{SO}_{4}$ and concentrated in vacuo. The residue was purified by column chromatography to give the compound YGP2 with $85 \%$ yield. HPLC (Agilent 1100; column/Extend-C18, $5 \mu \mathrm{m}, 4.6 \times 150 \mathrm{~mm}$; methanol:water/60:40; $0.6 \mathrm{ml} / \mathrm{min}),>99 \% ;{ }^{1} \mathrm{H} \mathrm{NMR}\left(\mathrm{CDCl}_{3}, 400 \mathrm{MHz}\right): \delta 8.65(\mathrm{~d}, 1 \mathrm{H})$, 8.38(dd, 1H), 8.28(m, 1H), $8.10(\mathrm{~s}, 1 \mathrm{H}), 7.92(\mathrm{~m}, 2 \mathrm{H}), 7.33(\mathrm{t}, 1 \mathrm{H})$, 7.16(dd, $2 \mathrm{H}) ;{ }^{13} \mathrm{C}$ NMR $\left(125 \mathrm{MHz}, \mathrm{CDCl}_{3}+\mathrm{CD}_{3} \mathrm{OD}\right): \delta 166.3$, $164.1,144.5,141.4,135.9,130.5,130.1 \times 2,128.6,124.0,115.6 \times$ 2. MS (ESI) m/z: $217[\mathrm{M}+\mathrm{H}]^{+}$; HRMS (ESI) 239.0597 Calcd for C12H9N2OFNa, found $239.0592[\mathrm{M}+\mathrm{Na}]^{+}$.

Structure characterization Compounds purchased from SPECS were purified before bioassay. The purity of the compounds listed in Figure $4 \mathrm{~B}$ has been determined by HPLC to be $\geq 95 \%$.

Homology modeling of the transmembrane domain of KCNQ2

KCNQ2 structures were modeled based on the structural information of the open-state Kv1.2 channel [29-31] by using Discovery studio 2.6 (Accelrys Inc., San Diego, CA, USA). Multiple se- quence alignment was generated by using CLUSTALW web server (http://www.ebi.ac.uk/Tools/msa/clustalw2) [48]. The highly conserved residues were used to guide the alignment (Supplementary information, Figure S1A), and the alignment was manually adjusted based on the transmembrane regions in the Kv1.2 structures [30, $31,49]$. The result of the sequence alignment is shown in Supplementary information, Figure S1A. The S4-S5 linker, S5, and S6 regions of Kv1.2 and KCNQ channels are highly conserved, which directly provides a reliable basis for mapping the positions of these regions of KCNQ channels based on the structure of Kv1.2 channel. The secondary structure and transmembrane domain were predicted by I-TASSER server [50] to guide the modeling of the S1S4 helix. Although residues are not so highly conserved in S1-S4 segments as those in S5 and S6 segments, the conserved residues, E0, E1, D3, R1, R2, R3, R4 and R5, provided enough information to locate the positions of S1-S4 (Supplementary information, Figure S1A). The conserved phenylalanine of S2 also helped to map the position of S2. As a result, the positions of S1-S4 could be accurately mapped. The modeled structure of KCNQ2 channels underwent a 20 000-step energy minimization in AMBER 10 [51].

\section{MD simulation}

MD simulations were performed on two systems - the KCNQ2 tetramer and the tetramer of the ztz240/KCNQ2 complex. MD simulation of KCNQ2 was started from the homology model of KCNQ2, and simulation of the complex started from the docking model of ztz240 to the KCNQ2 homology model. The starting structures of the three systems were embedded in a patch of POPC bilayer generated by using VMD [52], and then solvated by TIP3P water molecules [53]. MD simulation was carried out by using GROMACS 4.53 [54]. The CHARMM27 force field with CMAP correction was applied [55-57]; ztz240 parameters were generated by SWISSPARAM web server (http://www.swissparam.ch/) [58]. $\mathrm{Na}^{+}$and $\mathrm{Cl}^{-}$ions were added to maintain neutralization of the system. The NPT ensemble and 3D periodic boundary conditions were applied in the MD simulation, with the time step of $2 \mathrm{fs}$. The LINCS algorithm [59] was used to constrain the bond lengths. A constant pressure of 1 bar was applied with a coupling constant of 1.0 ps using Berendsen algorithm [60]. The v-rescale method [61] was employed to keep the temperature constant (300 K), with a coupling constant of 1.0 ps. The Particle-Mesh Ewald (PME) method [62] was used to handle the long-range electrostatics with a real-space cut-off of $1.2 \mathrm{~nm}$. The bilayer was first equilibrated to $10 \mathrm{~ns}$ with the protein and ztz240 fixed. The systems of KCNQ2 tetramer and ztz240/KCNQ2 complex were submitted to 50-ns and 200-ns production runs, respectively.

\section{Docking and virtual screening}

Docking was performed by using Glide (Schrödinger, LLC, New York, NY, USA). Receptor was prepared by using the Protein Preparation and Grid Preparation tools in the Schrödinger Maestro interface. Hydrogen orientations were optimized in protein preparation module. The docking box was set to cover the assumed binding pocket and centered at E130 and R207, which were supposed to be the middle of the potential pocket. The default settings were adopted for the cut-off, neutralization, scaling and dimension of the binding pocket. According to the primary mutagenesis and electrophysiological results, we noticed that ztz240 may access F137. Homology modeling could not predict proper conformations for the residues interacting with a ligand. Therefore, we applied 
the Induced Fit module encoded in the Schrödinger modeling package (Schrödinger, LLC). Inducted Fit is a flexible docking method that may consider the flexibilities of both the side chains of protein resides and small molecules during docking simulation. F137 and residues around F137 in the central part of VSD, E130, R207, S179, Q204, T133 and I134 were treated as flexible residues.

The modules of High Throughput Virtual Screening (HTVS) and Standard Precision (SP) were used as filters sequentially in the virtual screening. From the commercially available SPECS database, 20000 compounds were screened and the top $700 \mathrm{com}$ pounds with highest G-score values were inspected. Compounds with the docking positions showing high-energy conformations, such as unreasonable angles and torsions caused by the steric effect, were excluded. Among the remaining compounds, molecules with molecular weight $>500$ and those with large subgroups outside the pocket were also removed. The absorptions of the remaining potential hits were predicted by using Pipeline Pilot (Accelrys). AlogP $<5$ and Absorption_Level $=0$ were taken as filters. Finally, 25 compounds were selected as hits for bioassay.

\section{Volume calculation}

The sizes of the pockets in the KCNQ2 channel were calculated by using the program of POVME [63]. The sizes of ligands and the side chain of arginine were measured by the web server Molinspiration (http://www.molinspiration.com/).

\section{Data and statistical analysis}

Patch-clamp data were processed using Clampfit 9.2 (Molecular Devices), and then analyzed in Graphpad Prism 4 (GraphPad Software, San Diego, CA, USA). Voltage-dependent activation curves were fit with the Boltzmann equation: $G=G_{\min }+\left(G_{\max }-G_{\min }\right) /$ $\left.\left(1+\exp \left(V-V_{1 / 2}\right) / S\right)\right)$, where $G_{\max }$ is the maximum conductance, $G_{\min }$ is the minimum conductance, $V_{1 / 2}$ is the voltage for reaching $50 \%$ of maximum conductance and $S$ is the slope factor. Doseresponse curves were fit with the Hill equation: $E=E_{\max } /\left(1+\left(\mathrm{EC}_{50} /\right.\right.$ C) ${ }^{\mathrm{P}}$ ), where $\mathrm{EC}_{50}$ is the drug concentration producing half of the maximum response and $\mathrm{P}$ is the Hill coefficient. The deactivation traces were fit with exponential equations containing one or two components using Clampfit 9.2. Data are presented as means \pm SEM. Significance was estimated using paired two-tailed Student's $t$-tests.

\section{Acknowledgments}

We gratefully acknowledge the financial support from the State Key Development Program for Basic Research of China (2013CB910604 and 2009CB918502), the National Natural Science Foundation of China (81072579, 81173027, 21021063 and 81230076), Shanghai Science and Technology Development Funds (12QA1404000), and the National Institute of Health (MH084691 and GM070959). Computational resources were supported by National Supercomputing Center in Tianjin and Shanghai Supercomputer Center. We thank Professor Rolf Hilgenfeld (University of Lübeck, Germany) for his help in English language editing.

\section{References}

1 Hille B, eds. Ionic Channels of Excitable Membranes. 3rd
Edition, Sunderland: Sinauer, 2001.

2 Ashcroft FM, eds. Ion Channels and Diseases. New York: Academic Press, 1999.

3 Wulff H, Castle NA, Pardo LA. Voltage-gated potassium channels as therapeutic targets. Nat Rev Drug Discov 2009; 8:982-1001.

4 Chanda B, Asamoah OK, Blunck R, Roux B, Bezanilla F. Gating charge displacement in voltage-gated ion channels involves limited transmembrane movement. Nature 2005; 436:852856.

5 Jensen MO, Jogini V, Borhani DW, et al. Mechanism of voltage gating in potassium channels. Science 2012; 336:229-233.

6 Starace DM, Bezanilla F. Histidine scanning mutagenesis of basic residues of the $\mathrm{S} 4$ segment of the shaker $\mathrm{K}^{+}$channel. $J$ Gen Physiol 2001; 117:469-490.

7 Starace DM, Bezanilla F. A proton pore in a potassium channel voltage sensor reveals a focused electric field. Nature 2004; 427:548-553.

8 Larsson HP, Baker OS, Dhillon DS, Isacoff EY. Transmembrane movement of the shaker $\mathrm{K}^{+}$channel S4. Neuron 1996; 16:387-397.

9 Gandhi CS, Clark E, Loots E, Pralle A, Isacoff EY. The orientation and molecular movement of a $\mathrm{K}^{+}$channel voltagesensing domain. Neuron 2003; 40:515-525.

10 Goldstein SA. A structural vignette common to voltage sensors and conduction pores: canaliculi. Neuron 1996; 16:717722 .

11 Islas LD, Sigworth FJ. Electrostatics and the gating pore of Shaker potassium channels. J Gen Physiol 2001; 117:69-89.

12 Tombola F, Pathak MM, Gorostiza P, Isacoff EY. The twisted ion-permeation pathway of a resting voltage-sensing domain. Nature 2007; 445:546-549.

13 Tao X, Lee A, Limapichat W, Dougherty DA, MacKinnon R. A gating charge transfer center in voltage sensors. Science 2010; 328:67-73.

14 Starace DM, Stefani E, Bezanilla F. Voltage-dependent proton transport by the voltage sensor of the Shaker $\mathrm{K}^{+}$channel. Neuron 1997; 19:1319-1327.

15 Tombola F, Pathak MM, Isacoff EY. Voltage-sensing arginines in a potassium channel permeate and occlude cation-selective pores. Neuron 2005; 45:379-388.

16 Ahern CA, Horn R. Specificity of charge-carrying residues in the voltage sensor of potassium channels. J Gen Physiol 2004; 123:205-216.

17 Yang N, George AL Jr, Horn R. Molecular basis of charge movement in voltage-gated sodium channels. Neuron 1996; 16:113-122.

18 Baker OS, Larsson HP, Mannuzzu LM, Isacoff EY. Three transmembrane conformations and sequence-dependent displacement of the $\mathrm{S} 4$ domain in shaker $\mathrm{K}^{+}$channel gating. Neuron 1998; 20:1283-1294.

19 Padilla K, Wickenden AD, Gerlach AC, McCormack K. The KCNQ2/3 selective channel opener ICA-27243 binds to a novel voltage-sensor domain site. Neurosci Lett 2009; 465:138142.

20 Peretz A, Pell L, Gofman Y, et al. Targeting the voltage sensor of Kv7.2 voltage-gated $\mathrm{K}^{+}$channels with a new gating-modifier. Proc Natl Acad Sci USA 2010; 107:15637-15642.

21 Robbins J. KCNQ potassium channels: physiology, pathophy- 
siology, and pharmacology. Pharmacol Ther 2001; 90:1-19.

22 Xiong Q, Gao Z, Wang W, Li M. Activation of Kv7 (KCNQ) voltage-gated potassium channels by synthetic compounds. Trends Pharmacol Sci 2008; 29:99-107.

23 Gao Z, Zhang T, Wu M, et al. Isoform-specific prolongation of $\mathrm{Kv} 7$ (KCNQ) potassium channel opening mediated by new molecular determinants for drug-channel interactions. $J$ Biol Chem 2010; 285:28322-28332.

24 Stafstrom CE, Grippon S, Kirkpatrick P. Ezogabine (retigabine). Nat Rev Drug Discov 2011; 10:729-730.

25 Lee SY, Banerjee A, MacKinnon R. Two separate interfaces between the voltage sensor and pore are required for the function of voltage-dependent $\mathrm{K}^{+}$channels. PLoS Biol 2009; 7:e47.

26 Schenzer A, Friedrich T, Pusch M, et al. Molecular determinants of KCNQ (Kv7) $\mathrm{K}^{+}$channel sensitivity to the anticonvulsant retigabine. J Neurosci 2005; 25:5051-5060.

27 Wuttke TV, Seebohm G, Bail S, Maljevic S, Lerche H. The new anticonvulsant retigabine favors voltage-dependent opening of the Kv7.2 (KCNQ2) channel by binding to its activation gate. Mol Pharmacol 2005; 67:1009-1017.

28 Xiong Q, Sun H, Zhang Y, Nan F, Li M. Combinatorial augmentation of voltage-gated KCNQ potassium channels by chemical openers. Proc Natl Acad Sci USA 2008; 105:31283133.

29 Chen X, Wang Q, Ni F, Ma J. Structure of the full-length Shaker potassium channel Kv1.2 by normal-mode-based X-ray crystallographic refinement. Proc Natl Acad Sci USA 2010; 107:11352-11357.

30 Long SB, Campbell EB, Mackinnon R. Crystal structure of a mammalian voltage-dependent Shaker family $\mathrm{K}^{+}$channel. Science 2005; 309:897-903.

31 Pathak MM, Yarov-Yarovoy V, Agarwal G, et al. Closing in on the resting state of the Shaker $\mathrm{K}^{+}$channel. Neuron 2007; 56:124-140.

32 Kitchen DB, Decornez H, Furr JR, Bajorath J. Docking and scoring in virtual screening for drug discovery: methods and applications. Nat Rev Drug Discov 2004; 3:935-949.

33 Wickenden AD, Krajewski JL, London B, et al. N-(6-chloropyridin-3-yl)-3,4-difluoro-benzamide (ICA-27243): a novel, selective KCNQ2/Q3 potassium channel activator. Mol Pharmacol 2008; 73:977-986.

34 Hopkins AL, Groom CR. The druggable genome. Nat Rev Drug Discov 2002; 1:727-730.

35 Doyle DA, Morais Cabral J, Pfuetzner RA, et al. The structure of the potassium channel: molecular basis of $\mathrm{K}^{+}$conduction and selectivity. Science 1998; 280:69-77.

36 Jiang Y, Lee A, Chen J, et al. X-ray structure of a voltage-dependent $\mathrm{K}^{+}$channel. Nature 2003; 423:33-41.

37 Long SB, Campbell EB, Mackinnon R. Voltage sensor of Kv1.2: structural basis of electromechanical coupling. Science 2005; 309:903-908.

38 Perry M, Sachse FB, Sanguinetti MC. Structural basis of action for a human ether-a-go-go-related gene 1 potassium channel activator. Proc Natl Acad Sci USA 2007; 104:1382713832.

39 Perry M, Sanguinetti M, Mitcheson J. Revealing the structural basis of action of hERG potassium channel activators and blockers. J Physiol 2010; 588:3157-3167.

40 Xu X, Recanatini M, Roberti M, Tseng GN. Probing the bin- ding sites and mechanisms of action of two human ether-ago-go-related gene channel activators, 1,3-bis-(2-hydroxy5-trifluoromethyl-phenyl)-urea (NS1643) and 2-[2-(3,4-dichloro-phenyl)-2,3-dihydro-1H-isoindol-5-ylamino]-nicotinic acid (PD307243). Mol Pharmacol 2008; 73:1709-1721.

41 Perry M, Sachse FB, Abbruzzese J, Sanguinetti MC. PD118057 contacts the pore helix of hERG1 channels to attenuate inactivation and enhance $\mathrm{K}^{+}$conductance. Proc Natl Acad Sci USA 2009; 106:20075-20080.

42 Zhou PZ, Babcock J, Liu LQ, Li M, Gao ZB. Activation of human ether-a-go-go related gene (hERG) potassium channels by small molecules. Acta Pharmacol Sin 2011; 32:781-788.

43 Sasaki M, Takagi M, Okamura Y. A voltage sensor-domain protein is a voltage-gated proton channel. Science 2006; 312:589-592.

44 Ramsey IS, Moran MM, Chong JA, Clapham DE. A voltagegated proton-selective channel lacking the pore domain. $\mathrm{Na}$ ture 2006; 440:1213-1216.

45 Ramsey IS, Mokrab Y, Carvacho I, et al. An aqueous $\mathrm{H}^{+}$permeation pathway in the voltage-gated proton channel Hv1. Nat Struct Mol Biol 2010; 17:869-875.

46 Yarov-Yarovoy V, Baker D, Catterall WA. Voltage sensor conformations in the open and closed states in ROSETTA structural models of $\mathrm{K}^{+}$channels. Proc Natl Acad Sci USA 2006; 103:7292-7297.

47 Vargas E, Bezanilla F, Roux B. In search of a consensus model of the resting state of a voltage-sensing domain. Neuron 2011; 72:713-720.

48 Larkin MA, Blackshields G, Brown NP, et al. Clustal W and Clustal X version 2.0. Bioinformatics 2007; 23:2947-2948.

49 Long SB, Tao X, Campbell EB, MacKinnon R. Atomic structure of a voltage-dependent $\mathrm{K}^{+}$channel in a lipid membranelike environment. Nature 2007; 450:376-382.

50 Roy A, Kucukural A, Zhang Y. I-TASSER: a unified platform for automated protein structure and function prediction. Nat Protoc 2010; 5:725-738.

51 Case DA, Cheatham TE, Darden T, et al. The Amber biomolecular simulation programs. J Comput Chem 2005; 26:16681688 .

52 Humphrey W, Dalke A, Schulten K. VMD: visual molecular dynamics. J Mol Graph 1996; 14:33-38.

53 Jorgensen WL, Chandrasekhar J, Madura JD, Impey RW, Klein ML. Comparison of simple potential functions for simulating liquid water. $J$ Chem Phys 1983; 79:926-935.

54 Berendsen HJC, Vanderspoel D, Vandrunen R. Gromacs - a message-passing parallel molecular-dynamics implementation. Comput Phys Comm 1995; 91:43-56.

55 MacKerell AD, Bashford D, Bellott M, et al. All-atom empirical potential for molecular modeling and dynamics studies of proteins. J Phys Chem B 1998; 102:3586-3616.

56 MacKerell AD, Feig M, Brooks CL. Improved treatment of the protein backbone in empirical force fields. J Am Chem Soc 2004; 126:698-699.

57 Feller SE, MacKerell AD. An improved empirical potential energy function for molecular simulations of phospholipids. $J$ Phys Chem B 2000; 104:7510-7515.

58 Zoete V, Cuendet MA, Grosdidier A, Michielin O. SwissParam: a fast force field generation tool for small organic molecules. J Comput Chem 2011; 32:2359-2368. 
59 Hess B, Bekker H, Berendsen HJC, Fraaije JGEM. LINCS: A linear constraint solver for molecular simulations. $J$ Comput Chem 1997; 18:1463-1472.

60 Berendsen HJC, Postma JPM, Vangunsteren WF, Dinola A, Haak JR. Molecular-dynamics with coupling to an external bath. J Chem Phys 1984; 81:3684-3690.

61 Bussi G, Donadio D, Parrinello M. Canonical sampling through velocity rescaling. J Chem Phys 2007; 126:014101014107.

62 Darden T, York D, Pedersen L. Particle Mesh Ewald - an N.Log $(\mathrm{N})$ method for ewald sums in large systems. J Chem Phys 1993; 98:10089-10092.
63 Durrant JD, de Oliveira CA, McCammon JA. POVME: an algorithm for measuring binding-pocket volumes. J Mol Graph Model 2011; 29:773-776.

(Supplementary information is linked to the online version of the paper on the Cell Research website.)

(c) (i) (2) $($ This work is licensed under the Creative Commons Attribution-NonCommercial-No Derivative Works 3.0 Unported License. To view a copy of this license, visit http://creativecommons.org/licenses/by-nc-nd/3.0 